\title{
Using ranked auxiliary covariate as a more efficient sampling design for ANCOVA model: analysis of a psychological intervention to buttress resilience
}

\author{
Rajai Jabrah ${ }^{a}$, Hani M. Samawi ${ }^{1, a}$, Robert Vogel $^{a}$, Haresh D. Rochani ${ }^{a}$, \\ Daniel F. Linder ${ }^{b}$, Jeff Klibert ${ }^{c}$ \\ ${ }^{a}$ Department of Biostatistics, Jiann-Ping Hsu College Public Health, Georgia Southern \\ University, USA; ${ }^{b}$ Department of Biostatistics and Epidemiology, Augusta University, USA; \\ ${ }^{c}$ Psychology Department, Georgia Southern University, USA
}

\begin{abstract}
Drawing a sample can be costly or time consuming in some studies. However, it may be possible to rank the sampling units according to some baseline auxiliary covariates, which are easily obtainable, and/or cost efficient. Ranked set sampling (RSS) is a method to achieve this goal. In this paper, we propose a modified approach of the RSS method to allocate units into an experimental study that compares $L$ groups. Computer simulation estimates the empirical nominal values and the empirical power values for the test procedure of comparing $L$ different groups using modified RSS based on the regression approach in analysis of covariance (ANCOVA) models. A comparison to simple random sampling (SRS) is made to demonstrate efficiency. The results indicate that the required sample sizes for a given precision are smaller under RSS than under SRS. The modified RSS protocol was applied to an experimental study. The experimental study was designed to obtain a better understanding of the pathways by which positive experiences (i.e., goal completion) contribute to higher levels of happiness, well-being, and life satisfaction. The use of the RSS method resulted in a cost reduction associated with smaller sample size without losing the precision of the analysis.
\end{abstract}

Keywords: ranked set sampling, ranked auxiliary covariate, sampling design, cost-effectiveness, reduced sample size, empirical power, emotional uplifting intervention, ANCOVA, multiple regression

\section{Introduction}

Sufficient funding and time is a frequent challenge for researchers attempting to draw accurate outcomes from the sample of the population under study. Therefore, the sampling design is a critical element of the research protocol (Sandelowski, 2000). Some areas of research (such as: ecological, environmental, agricultural, and epidemiological studies) contain situations where the measurement of the response variable is costly or time consuming. However, other information such as an auxiliary covariate that is correlated to the variable of interest can be easily and economically obtained. The auxiliary covariate can be used to assist in selecting a more efficient sample and reduce the cost of the study (Chen et al., 2004).

\footnotetext{
${ }^{1}$ Corresponding author: Department of Biostatistics, Karl Peace Center for Biostatistics, Jiann-Ping Hsu College Public Health, Georgia Southern University, P.O. Box 8015, Statesboro, GA 30460, USA. E-mail: hsamawi@georgiasouthern.edu
}

Published 31 May 2017 / journal homepage: http://csam.or.kr

(c) 2017 The Korean Statistical Society, and Korean International Statistical Society. All rights reserved. 
Ranked set sampling (RSS) was proposed by McIntyre (1952) and has been used extensively in environmental and ecological studies as an alternative method to simple random sampling (SRS). RSS was designed to estimate the mean with improved efficiency. When applying RSS, it is assumed that a sample from a population can be inexpensively and easily ordered by direct ranking methods such as visual inspection, or by indirect ranking methods based on an auxiliary covariate that is highly correlated with the variable of interest. Along with the outcome of interest, other information such as demographic and physiological characteristics (including age, gender, weight, and blood pressure measures) might be recorded. These are called "auxiliary covariates." Some of these auxiliary covariates can be highly correlated to the outcome of interest. Therefore, the information contained in covariates can assist in subject selection for the sample as well as to improve inference efficiency when comparing $L$ groups (Chen et al., 2008). Egger et al. (1985) suggested that hypothesis testing could be improved by incorporating baseline information into the model under study. Donner and Zou (2007) showed that the efficiency of treatment comparisons could be improved if highly correlated baseline measurements and outcome measurements were accounted for in the statistical analysis. Tsiatis $e t$ al. (2008) proposed an approach of adjustment for auxiliary covariates to improve the inference of randomized clinical trials. Zhang et al. (2008) also proposed an approach to adjust for auxiliary covariates to improve the precision of estimating treatment effects and the general null hypothesis in the analysis of randomized clinical trials by using semi-parametric theory. All researchers showed that the use of auxiliary covariates related to the outcome of interest could improve efficiency.

The RSS technique is an efficient alternative to the SRS method in agricultural and environmental studies that can also be used as an efficient means to incorporate auxiliary information at the design stage of human studies (Samawi and Vogel, 2015). RSS has gained attention and application in recent years by researchers conducting clinical studies who use available data from medical records that are correlated to the medical measure under consideration. RSS can be used by ranking the selected random sample according to the data in those medical records.

Stokes (1977) introduced the use of RSS in the linear regression model. He showed that RSS would have been a better choice than SRS to estimate the population mean by modeling the relation between auxiliary variables and the variable of interest for the linear regression model. In addition, research shows that the RSS provides unbiased and more efficient parameter estimation as well as powerful testing compared to those based on SRS method (Samawi and Abu-Dayyeh, 2002). Muttlak (1996) considered RSS for simple and multiple regression models. Under his model assumptions, no improvement made in estimating the parameters. Barreto and Barnett (1999) considered a simple linear regression model with replicated observations obtained from RSS. They considered a case where the dependent variable was normally distributed and ranking was assumed to be perfect. The estimators of slope and intercept parameters based on RSS were proven more efficient than the estimators based on SRS. Samawi and Ababneh (2001) used RSS, ranking only on the variable $(X)$, to investigate the effect of RSS on regression analysis in general. They found RSS more efficient than SRS for estimating model parameters (Patil et al., 1993). Samawi and Abu-Dayyeh (2002) demonstrated that extreme ranked set sampling also improved the performance of estimating the model parameters. Özdemir and Esin (2007) also examined the RSS method in the simple and the multiple linear regression model. They concluded that RSS provided a more efficient way to conduct a regression analysis. However, to assess the relation between the outcome $(Y)$ and risk factors and/or covariates $(X)$ in different groups, complex models such as multiple linear regression and analysis of variance (ANOVA) have received little attention (Muttlak, 1996).

Consequently, the idea proposed in this paper is to improve inference when comparing groups by incorporating ranked auxiliary covariates that are correlated to the variable of interest. To use 
this method effectively, we have the additional assumption that the response variable is difficult and expensive to obtain. To implement this proposal, a random sampling process is applied to a large cohort by using an RSS technique based on inexpensive auxiliary covariates and correlated with the outcome of interest. We propose that our approach will provide a more precise estimator of the mean of the outcome of interest.

In the next section, we will focus on the use of RSS in more complex models to select sampling units and their assignment to $L$ groups. The mean of the response variable for the groups will be estimated in analysis of covariance (ANCOVA) models.

\section{Analysis of covariance (ANCOVA) using ranked set sampling (RSS)}

We propose a parametric method for inference by using the multiple regression approach to the ANCOVA model to compare the means of $L$ treatments. We will explore the properties of the ANCOVA under the modified RSS design.

The two purposes of the ANCOVA models are to increase the precision of comparisons between groups by accounting to variation on important auxiliary variables and to "adjust" comparisons between groups for imbalances in important auxiliary variables between these groups. For this to work properly, the choice of auxiliary covariates is important. There is no gain by using ANCOVA if a covariate has no relation to the response variable; therefore, we propose to use an auxiliary covariate that has high correlation to the response variable.

\subsection{Modified RSS for ANCOVA model}

Assume the sampling units drawn from the population can be economically ranked. For example, given experimental conditions, the procedure of selecting a RSS involves selecting simple random samples of subjects each of size $m$, where $m$ is called a set size. The process of the modified RSS scheme is to allocate subjects to the $L$ different groups as:

Step 1. Randomly select $(L)$ independent sets each containing $(m)$ subjects identified from a population or a cohort.

Step 2. Within each selected sample set, rank the subjects with respect to the auxiliary baseline covariate $(X)$ which is correlated with the response variable $(Y)$.

Step 3. From each set of selected subjects, the subject with minimum value of $(X)$ will be selected for study. Different experimental conditions $(L)$ will be assigned randomly to the selected subjects.

Step 4. The remaining $L(m-1)$ subjects from the selected set will not be selected for measurement of outcome $(Y)$. Repeat Steps 1-3. However, in Step 3, subjects with second minimum rank of the value of $(X)$ will be selected for the study. The experimental conditions will be randomly assigned to the $(L)$ selected subjects.

Step 5. The process will continue in the same way until the subjects with rank $m$ of $(X)$ values are selected and randomly assigned to one of the $(L)$ experimental conditions.

The above process represents a single RSS cycle for each experimental condition. The total sample size of the selected subjects is $L m r$. In term of notations, let $X_{i(1) k}, X_{i(2) k}, X_{i(m) k}$, where $i=1, \ldots, L$ experimental conditions, and $k=1, \ldots, r$ cycles, denote a random sample of subjects of size $L m r$ 
based on the auxiliary variable $(X)$. Then, $X_{i(j) k}$ is the $j^{\text {th }}$ ranked subject, from the $i^{\text {th }}$ experimental condition in the $k^{t h}$ cycle. Therefore, $\left\{\left(X_{i(j) k}, Y_{i[j] k}\right), i=1,2, \ldots, L ; J=1, \ldots, m ; k=1,2, \ldots, r\right\}$, will denote the quantified data to be analyzed using the ANCOVA model.

\subsection{ANCOVA models to compare $L$ groups for RSS}

Let the outcome variable of interest $(Y)$ be a random variable with p.d.f. $f_{Y}(y)$ with a finite mean $\mu_{Y}$, and a finite variance $\sigma_{Y}^{2}$. Let $\left\{\left(Y_{i[1] 1}, X_{i(1) 1}, Z_{i}\right), \ldots,\left(Y_{i[m] 1}, X_{i(m) 1}, Z_{i}\right) ; \ldots ;\left(Y_{i[1] r}, X_{i(1) r}, Z_{i}\right), \ldots,\left(Y_{i[m] r}\right.\right.$, $\left.\left.X_{i(m) r}, Z_{i}\right)\right\}$ be the selected RSS of size $m$ and cycle size $r$ assigned to $i^{\text {th }}, i=1,2, \ldots, L$ condition group, where $(X)$ is the auxiliary covariate $(Z)$ is the indicator variable the experiment $(Z)$. Therefore, for the $i^{\text {th }}$ treatment the linear model can be written as

$$
y_{i[j] k}=\mu_{0}+\beta_{1} x_{i(j) k}+\gamma_{i} Z_{i}+\epsilon_{i[j] k}, \quad i=1,2, \ldots, L ; J=1,2, \ldots, m ; k=1,2, \ldots, r,
$$

where $[j]$ denote the imperfect $j^{\text {th }}$ ranking (ranking with errors with respect to the response variable $Y) . \mu_{0}$ is the overall mean, $\beta_{1}$ is the slope of $(X), \gamma_{i}$ is the $i^{\text {th }}$ experiment group, and $\epsilon_{i(j) k}$ is the random error. In matrix notation, (2.1) can be written by

$$
Y_{R}=\beta X_{R}+\gamma Z_{R}+\epsilon_{R},
$$

$\gamma=\left[\gamma_{1}, \gamma_{2}, \ldots, \gamma_{L-1}\right]^{\prime}$ is the vector of $L-1$ experiment groups effect, and $\epsilon$ is a vector of the random error term. Note that $\gamma_{L}=\left[-\gamma_{1},-\gamma_{2}, \ldots,-\gamma_{L-1}\right]^{\prime}$. Also, let

$$
Z_{R}=\left[I_{1}, I_{2}, \ldots, I_{L-1}\right],
$$

where,

$$
\begin{gathered}
I_{1}=\left\{\begin{aligned}
1, & \text { if case from treatment } 1, \\
-1, & \text { if case from treatment } L, \\
0, & \text { otherwise, }
\end{aligned}\right. \\
I_{L-1}=\left\{\begin{aligned}
1, & \text { if case from treatment } L-1, \\
-1, & \text { if case from treatment } L, \\
0, & \text { otherwise }
\end{aligned}\right.
\end{gathered}
$$

are experimental condition indicators. Therefore, the model (2.1) can be expressed as follows:

$$
y_{i[j] k}=\beta_{0}+\beta_{1} x_{i(j) k}+\gamma_{1} I_{1}+\cdots+\gamma_{L-1} I_{L-1}+\epsilon_{i[j] k}
$$

where $\epsilon_{i[j] k}$ is independent and normally distributed as $\epsilon_{i[j] k} \sim N\left(0, \sigma_{[j]}^{2}\right)$.

Since model (2.2) contains an intercept then, the design matrix $(X)$ has a column of ones and can be written as $\left[I_{n}, x\right]$, and $x$ denotes the centered observation $\left(x_{i(j) k}-\bar{x}_{i(j)}\right)$, where $\bar{x}_{i(j)}=\sum_{k=1}^{r} \bar{x}_{i(j) k} / r$, $i=1,2, \ldots, L ; j \stackrel{\sim}{=} 1,2, \ldots, m$.

In the next section, we will develop theoretical aspects needed to analyze the ranked based data in ANCOVA models. 


\subsubsection{Some asymptotic results of using the modified RSS}

If subjects are selected into group $i$ based on a RSS judgment, which then leads to the $j^{\text {th }}$ order statistics, the random variable $Y_{i[j] k}$ has variance $\sigma_{Y_{[j]}}^{2}$. The differences between the expected mean of order statistics and population mean play an important role in RSS method because $\sigma_{Y_{[j]}}^{2}=\sigma_{Y}^{2}-\Delta_{Y_{[j]}}$, where $\Delta_{Y_{[j]}}=\left(\mu_{Y_{[j]}}-\mu_{Y}\right)^{2}$. Let $Y_{i[j] 1}, \ldots, Y_{i[j] r}$ be an independent random sample of size $(r)$ of the $j^{\text {th }}$ order statistics in experimental group, then the sample mean is $\bar{Y}_{[j]}=1 / r \sum_{k=1}^{r} Y_{[j] k}$. Therefore, $E\left[\bar{Y}_{[j]}\right]=\mu_{Y_{[j]}}$ and $V\left(\bar{Y}_{[j]}\right)=\sigma_{Y_{[j]}}^{2} / r$.

To show the asymptotic properties of the model's parameter estimates in the ANCOVA model, note that the $k^{\text {th }}$ moment for the ordered statistics exist if and only if the $k^{\text {th }}$ moment of the original population's outcome exists (Rohatgi and Ehsanes Saleh, 2001, p. 177).

Theorem 1. Let $Y_{i[j] 1}, Y_{i[j] 2}, \ldots, Y_{i[j] r}$ be a random sample from a population with $c d f F_{Y_{[j]}}$, pdf $f_{\left.Y_{[j]}\right]}$, variance $V\left(Y_{[j]}\right)=\sigma_{Y_{[j]}}^{2}$ and finite fourth moment $\left(\mu_{4[j]}\right)$ where $Y_{[j]}$ is the $j^{\text {th }}$ order statistics of a SRS of size $m$. Then the sample variance $S_{Y_{[j]}}^{2}$ is a consistent estimator of the population variance $\sigma_{Y_{[j]}}^{2}$.

Proof: The proof is a consequence of $\bar{Y}_{[j]} \stackrel{P}{\rightarrow} \mu_{Y_{j}}$ by the WLLN , and since $S_{Y_{[j]}}^{2}$ is an unbiased estimator and $V\left(S_{Y_{[j]}}^{2}\right)=\mu_{4[j]} / r+\left((3-r) \mu_{2[j]}^{2}\right) / r(r-1) \rightarrow 0$ for large $r$. Using the above results, we derive the estimator of the ANCOVA model parameters using the ranked auxiliary covariate to compare between $L$ groups.

\subsubsection{Estimating unknown parameters: weighted least squares estimation (WLS)}

We can rewrite the model (2.2) as,

$$
Y_{R}=M_{R} \theta+\epsilon_{R},
$$

where $M_{R}=\left[\begin{array}{ll}X_{R} & Z_{R}\end{array}\right]^{\prime}, \theta=\left[\begin{array}{ll}\beta & \gamma\end{array}\right]^{\prime}$ and $\epsilon_{R}$ is a random error term.

The method of ordinary least squares (OLS) is not recommended for estimation in this case because the variance of $Y_{[j]}$ is not the same for all $j$ 's. Therefore, WLS method is recommended as an alternative to OLS when stabilizing variance. Given that, $V\left(Y_{[j]}\right)=\sigma_{Y_{[j]}}^{2}$, let $\Sigma$ be the variancecovariance matrix of $Y_{R}$. The most common and straightforward weighting scheme is $w_{j}=1 / \sigma_{Y_{[j]}}$. However, $\sigma_{Y_{[j]}}^{2}$ is unknown, so the estimated weight is given by, $\hat{w}_{j}=1 / S_{Y_{[j]}}$. The diagonal of the estimated weighting coefficient matrix is denoted by $\widehat{W}=\hat{\Sigma}^{-1 / 2}$ and we rewrite model (2.6) as

$$
\widehat{W} Y_{R}=\widehat{W} M_{R} \theta+\widehat{W} \epsilon_{R}
$$

The model in (2.7) can be written as,

$$
V=R \theta+\epsilon^{*}
$$

where $V=\widehat{W} Y_{R}, R=\widehat{W} M_{R}$ and $\epsilon^{*}=\widehat{W} \epsilon_{R}$

The vector of auxiliary covariates and group indicator $(M)$ is assumed to be known and available for a finite number of parameters $\theta=\left(\theta_{1}, \ldots, \theta_{p-1}\right)^{\prime}$, where $p<n$. We estimate the parameter $\theta$ by the value $\hat{\theta}$ that gives the best fit to the model. The WLS, denoted by $\hat{\theta}$, is that value of $\theta$ that minimizes the sum square of errors. Denote the squared length of an $n$-dimensional vector $\epsilon$ by $\left\|\epsilon^{*}\right\|^{2}=\epsilon^{* \prime} \epsilon$. Then it is easy to see that in weighted least squares, we are minimizing,

$$
\epsilon^{* \prime} \epsilon=(V-R \theta)^{\prime}(V-R \theta),
$$


given that $\hat{\theta}=\left(R^{\prime} R\right)^{-1} R^{\prime} V$. To find the values of errors sum of squares (SSE), parameter estimator of $\hat{\theta}$ includes the ranked auxiliary covariates effects $\hat{\beta}$ and treatment effects $\hat{\gamma}$, we will use the sweeping operator method.

Let $C=\widehat{W}^{\prime} \widehat{W} \cong W^{\prime} W$, then for the reduced model,

$$
\operatorname{SSE}_{\text {(Reduced) }}\left(X_{R}\right)=Y_{R}^{\prime} \widehat{W}^{\prime}(I-H) \widehat{W} Y_{R}
$$

and

$$
\hat{\beta}=\left(X_{R}^{\prime} C X_{R}\right)^{-1} X_{R}^{\prime} C Y_{R}
$$

For the full model,

$$
\operatorname{SSE}_{(\mathrm{Full})}\left(X_{R}, Z_{R}\right)=Y_{R}^{\prime} \widehat{W}^{\prime}(I-H) \widehat{W} Y_{R}-Y_{R}^{\prime} \widehat{W}^{\prime}(I-H) \widehat{W} Z_{R}\left(Z_{R}^{\prime} \widehat{W}^{\prime}(I-H) \widehat{W} Z_{R}\right)^{-1} Z_{R}^{\prime} \widehat{W}^{\prime}(I-H) \widehat{W} Y_{R}
$$

and the estimated unknown parameters are given by,

$$
\hat{\gamma}_{\mathrm{RSS}}=\left(Z_{R}^{\prime} \widehat{W}^{\prime}(I-H) \widehat{W} Z_{R}\right)^{-1} Z_{R}^{\prime} \widehat{W}^{\prime}(I-H) \widehat{W} Y_{R}
$$

and

$$
\begin{aligned}
\hat{\beta}_{\mathrm{RSS}} & =\left(X_{R}^{\prime} C X_{R}\right)^{-1} X_{R}^{\prime} C Y_{R}-\left(X_{R}^{\prime} C X_{R}\right)^{-1} X_{R}^{\prime} C Z_{R}\left(Z_{R}^{\prime} \widehat{W}^{\prime}(I-H) \hat{W} Z_{R}\right)^{-1} Z_{R}^{\prime} \widehat{W}^{\prime}(I-H) \widehat{W} Y_{R} \\
& =\hat{\beta}_{\text {(Reduced) }}-\left(X_{R}^{\prime} C X_{R}\right)^{-1} X_{R}^{\prime} C Z_{R} \hat{\gamma}_{(\mathrm{RSS})},
\end{aligned}
$$

where $H=\widehat{W} X_{R}\left(X_{R}^{\prime} C X_{R}\right)^{-1} X_{R}^{\prime} \widehat{W}^{\prime}$. The estimated parameters $\hat{\beta}_{\mathrm{RSS}}$ and $\hat{\gamma}_{\mathrm{RSS}}$ are unbiased estimators for $\beta$ and $\gamma$, as showing in the following theorem.

Theorem 2. Let $\widehat{W} Y_{R}=\widehat{W} X_{R} \beta+\widehat{W} Z_{R} \gamma+\widehat{W} \epsilon_{R}$, where $X_{R}$ is $n L \times p, Z_{R}$ is a $n L \times(L-1)$ matrices of full rank; $\beta$ and $\gamma$ are $[p \times 1]$ and $[(L-1) \times 1]$, respectively vectors of unknown parameters; $\epsilon$ is an $n \times 1$ random errors vector with mean and variance defined by order statistics $\left(0, \sigma_{Y_{[j]}}^{2}\right)$. The WLS $\hat{\beta}_{R S S}$ and $\hat{\gamma}_{R S S}$ are unbiased estimators for $\beta$ and $\gamma$, respectively.

Proof: Applying Theorem 1, for the large sample size we can write the estimated weighted variancecovariance matrix $\widehat{W} \cong W^{\prime}=\Sigma^{-1 / 2}$, then we show that the expected value of $\hat{\beta}_{\mathrm{RSS}}$ is given by,

$$
\begin{aligned}
E\left(\hat{\beta}_{\mathrm{RSS}}\right) & =\left[1\left(X_{R}^{\prime} C X_{R}\right)^{-1} X_{R}^{\prime} C-\left(X_{R}^{\prime} C X_{R}\right)^{-1} X_{R}^{\prime} C Z_{R}\left(Z_{R}^{\prime} C W^{\prime}(I-H) W Z_{R}\right)^{-1} Z_{R}^{\prime} W^{\prime}(I-H) W\right] E\left(Y_{R}\right) \\
& =\beta-0+\left(X_{R}^{\prime} C X_{R}\right)^{-1} X_{R}^{\prime} C Z_{R} \gamma-\left(X_{R}^{\prime} C X_{R}\right)^{-1} X_{R}^{\prime} C Z_{R} \gamma=\beta
\end{aligned}
$$

and

$$
\begin{aligned}
E\left(\hat{\gamma}_{\mathrm{RSS}}\right) & =\left[\left(Z_{R}^{\prime} W^{\prime}(I-H) W^{\prime} Z_{R}\right)^{-1} Z_{R}^{\prime} W^{\prime}(I-H)\right] E\left(Y_{R}\right) \\
& =\left(Z_{R}^{\prime} W^{\prime}(I-H) W Z_{R}\right)^{-1} Z_{R}^{\prime} W^{\prime}(I-H) W X_{R} \beta+\left(Z_{R}^{\prime} W^{\prime}(I-H) W Z_{R}\right)^{-1} Z_{R}^{\prime} W^{\prime}(I-H) W Z_{R} \gamma \\
& =\gamma .
\end{aligned}
$$


Now, since $V\left(W Y_{R}\right)=W^{\prime} \Sigma W=I$, the variances of the estimators of the unknown parameters are given by, $V\left(\hat{\beta}_{(\mathrm{RSS})}\right)=\left(X_{R}^{\prime} C X_{R}\right)^{-1}+\left(X_{R}^{\prime} C X_{R}\right)^{-1} X_{R}^{\prime} C Z_{R}\left(Z_{R}^{\prime} W^{\prime}(I-H) W Z_{R}\right)^{-1} Z_{R}^{\prime} C X_{R}\left(X_{R}^{\prime} C X_{R}\right)^{-1}$ and $V\left(\hat{\gamma}_{\mathrm{RSS}}\right)=\left[\left(Z_{R}^{\prime} W^{\prime}(I-H) W Z_{R}\right)^{-1}\right]$.

When testing the null hypothesis $H_{0}: \gamma_{1}=\gamma_{2}=\cdots=\gamma_{L}=0$, it can be shown that, for the reduced model $\widehat{W} Y=\widehat{W} X_{R} \beta+\widehat{W} \epsilon$, where $X_{R}$ is of rank $n p . E(\epsilon)=0$ and $V(\epsilon)=\Sigma, s_{1}^{2}=$ $\left(\operatorname{SSE}_{\text {(Reduced) }}\left(X_{R}\right)\right) /(n L-p)$ is an unbiased estimator for $W^{\prime} \Sigma W$. Under the normality assumption of $Y$, it can be shown that $\operatorname{SSE}_{\text {(Reduced) }}\left(X_{R}\right)$ follows a chi-square distribution with $(n L-p)$ degrees of freedom. Similarly, in the full model, $s_{2}^{2}$ is given by, $s_{2}^{2}=\operatorname{SSE}_{(\mathrm{Full})}\left(X_{R}, Z_{R}\right) n L-p-L+1$ is unbiased estimator for $W^{\prime} \Sigma W$. Under the normality assumption of $Y, \mathrm{SSE}_{(\mathrm{Full})}\left(X_{R}, Z_{R}\right)$ approximately follows a chi-square distribution with $(n L-p-L+1)$ degrees of freedom. Using the above results, the differences between the reduced model SSE, $\operatorname{SSE}_{\text {(Reduced) }}\left(X_{R}\right)$, and the full model SSE, $\operatorname{SSE}_{(\text {Full) }}\left(X_{R}, Z_{R}\right)$, is given by

$$
\begin{aligned}
\operatorname{SSR}\left(Z_{R} \mid X_{R}\right) & =\operatorname{SSE}_{\text {Reduced }}\left(X_{R}\right)-\operatorname{SSE}_{\text {Full }}\left(X_{R}, Z_{R}\right) \\
& =Y^{\prime} A Z_{R}\left(Z_{R}^{\prime} A Z_{R}\right)^{-1} Z_{R}^{\prime} A Y_{R},
\end{aligned}
$$

and under the null hypothesis $H_{0}: \gamma_{1}=\gamma_{2}=\cdots=\gamma_{L}=0, \operatorname{SSR}\left(Z_{R} \mid X_{R}\right)$ is an unbiased estimator $W^{\prime} \Sigma W$. Hence, under the null hypothesis $\operatorname{SSR}\left(Z_{R} \mid X_{R}\right)$ approximately follows a chi-square distribution with $L-1$ degrees of freedom.

For the fixed effect model (2.1), the hypothesis testing for treatment effects involve testing the hypotheses, $H_{0}: \gamma_{1}=\gamma_{2}=\cdots=\gamma_{L}=0$, as:

$$
\begin{aligned}
F^{*} & =\frac{\operatorname{SSE}(R)-\operatorname{SSE}(F)}{\mathrm{df}_{R}-\mathrm{df}_{F}} \div \frac{\operatorname{SSE}(F)}{\mathrm{df}_{F}} \\
& =\frac{\operatorname{SSR}\left(Z_{R} \mid X_{R}\right)(L-1)}{\operatorname{SSE}(F) n L-p-L+1}
\end{aligned}
$$

which has approximately $F$-distribution with $(L-1, n L-p-L+1)$ degrees of freedom.

\section{Simulation study}

A simulation study was conducted to investigate the performance of the modified RSS method when applied to $L$ groups for comparison to SRS under ANCOVA. In this simulation study, the empirical nominal value $(\alpha)$ and the empirical power values are estimated for the modified RSS and compared to the SRS. The auxiliary covariate $(X)$ is assumed to be a continuous variable, and it is ranked based on an order statistics method. For each group, samples were selected by set size of ( $m=3,4$, and 5) units and cycles $(k=10$ and 30). For accurate comparison, the samples of RSS and SRS, within each group were selected with the same sample sizes, $(n=30,40,50,90,120$, and 150), where $n=L m r$. The level of significant was set at $\alpha=0.05$ is considered. Different correlation coefficients between the auxiliary covariate $(X)$ and the variable of interest $(Y)$ were set at $(\rho \pm 0.3, \pm 0.5, \pm 0.8)$. The differences in means between groups $(d=0,0.3,0.5,0.8)$ were used for two experimental groups; however, the differences in means of $\left(d_{1}=0,0.3,0.5,0.8\right)$ and $\left(d_{2}=0,0.3,1\right)$ were used for three experimental groups. The normal distribution is used in the simulation, with all simulations are based on 5,000 replicates.

Table 1 presents the estimated probability of the type I error under the null hypothesis of no differences between the means of two groups, based on RSS and SRS. In general, both sampling 
Table 1: Empirical nominal value $(\alpha=0.05)$ for two interventional groups

\begin{tabular}{|c|c|c|c|c|c|c|c|}
\hline \multirow[b]{2}{*}{$r$} & \multirow[b]{2}{*}{$\rho$} & \multicolumn{2}{|c|}{$m=3$} & \multicolumn{2}{|c|}{$m=4$} & \multicolumn{2}{|c|}{$m=5$} \\
\hline & & RSS & SRS & RSS & SRS & RSS & SRS \\
\hline 10 & & 0.0410 & 0.0542 & 0.0474 & 0.0498 & 0.0462 & 0.0494 \\
\hline 30 & 0.3 & 0.0340 & 0.0490 & 0.0337 & 0.0483 & 0.0376 & 0.0536 \\
\hline 10 & & 0.0412 & 0.0476 & 0.0444 & 0.0524 & 0.0448 & 0.0448 \\
\hline 30 & 0.5 & 0.0410 & 0.0494 & 0.0410 & 0.0494 & 0.0366 & 0.0448 \\
\hline 10 & & 0.0388 & 0.0472 & 0.0496 & 0.0562 & 0.0478 & 0.0542 \\
\hline 30 & 0.9 & 0.0418 & 0.0452 & 0.0442 & 0.055 & 0.0440 & 0.0484 \\
\hline 10 & & 0.0316 & 0.0362 & 0.0441 & 0.0478 & 0.0475 & 0.0528 \\
\hline 30 & -0.3 & 0.0378 & 0.0482 & 0.0402 & 0.0503 & 0.0414 & 0.0452 \\
\hline 10 & & 0.0404 & 0.0496 & 0.0390 & 0.0564 & 0.0440 & 0.0476 \\
\hline 30 & -0.5 & 0.0418 & 0.0470 & 0.0424 & 0.0522 & 0.0442 & 0.0470 \\
\hline 10 & & 0.0500 & 0.0508 & 0.0458 & 0.0524 & 0.0544 & 0.0558 \\
\hline 30 & -0.9 & 0.0390 & 0.0498 & 0.0384 & 0.0536 & 0.0398 & 0.0510 \\
\hline
\end{tabular}

RSS = ranked set sampling; SRS = simple random sampling

techniques RSS and SRS give close estimates to the nominal value of $\alpha(\alpha=0.05)$ for all suggested set sizes, sample sizes, and correlation coefficient values. However, when using RSS in ANCOVA it is clear that the estimated nominal value is smaller than SRS for different sample sizes and different correlation coefficients.

Table 2 shows the estimated empirical power under different alternative hypotheses when comparing two experimental groups. In general, these tables show that using RSS scheme is more powerful than using SRS method for all given correlation coefficients. The power also increases as the mean difference of the two groups $(d)$, sample size, and correlation coefficient increases. However, the results are more powerful for positive correlation coefficients than the negative for both sampling schemes. Similarly, Table 3 shows the empirical nominal value of for the mean differences between three experimental groups, using RSS and SRS. In general, both sampling schemes of RSS and SRS give close estimates to the nominal value of $(\alpha=0.05)$ for all suggested set sizes, sample sizes, and correlation coefficient values. However, results show that the nominal value $(\alpha)$ for the modified RSS is smaller than the SRS for different sample sizes and different correlation coefficients.

Table 4 shows the empirical power under different alternative hypotheses when comparing three groups. Table 4 often shows that the use of a RSS scheme results in a more powerful testing procedure than the use of a SRS method for all given correlation coefficients. In addition, the power increases as the mean differences $\left(d_{1}, d_{2}\right)$, sample size, and correlation coefficient increases when using RSS in the regression model to compare the effect of the groups.

Finally, we include small scale simulation study to compare our proposed approach with the ordinary regression analysis using RSS. In the ordinary regression approach, the indicator variables used for the treatment groups variable (treatment group $=1$, no treatment group $=0$ ), and then the ordinary least square estimate (OLSE) method is used. Table 5 shows that our proposed approach adjusting for the probability of type I error $(\alpha=0.05)$ while the OLSE regression approach failed to adjust for $\alpha$ This may be because the OLSE method assumes equal variances across all groups. However, $\operatorname{Var}\left(Y_{[j]}\right)=\sigma_{[j]}^{2} \neq \sigma^{2}$ for $j=1,2, \ldots, m$. Therefore, our approach provides better analysis to control for $\alpha$ and improve the power when comparing treatment groups.

\section{Boosting happiness and buttressing resilience: uplifting intervention designed by using ranked set sampling (RSS) approach}

The following study was conducted to obtain a better understanding of the pathways by which positive experiences (i.e., goal completion) contribute to higher levels of happiness, well-being, and life satisfaction. Undergraduate students at Georgia Southern University were the study population for 
Table 2: Empirical power for testing two interventional groups with the mean groups different $(d)$

\begin{tabular}{|c|c|c|c|c|c|c|c|c|}
\hline \multirow{2}{*}{$d$} & \multirow{2}{*}{$r$} & \multirow{2}{*}{$\rho$} & \multicolumn{2}{|c|}{$m=3$} & \multicolumn{2}{|c|}{$m=4$} & \multicolumn{2}{|c|}{$m=5$} \\
\hline & & & RSS & SRS & RSS & SRS & RSS & SRS \\
\hline \multirow{6}{*}{0.3} & $\begin{array}{l}10 \\
30\end{array}$ & 0.3 & $\begin{array}{l}0.0692 \\
0.1080\end{array}$ & $\begin{array}{l}0.0690 \\
0.1072\end{array}$ & $\begin{array}{l}0.0892 \\
0.1530\end{array}$ & $\begin{array}{l}0.0760 \\
0.1258\end{array}$ & $\begin{array}{l}0.1048 \\
0.2124\end{array}$ & $\begin{array}{l}0.0788 \\
0.1460\end{array}$ \\
\hline & $\begin{array}{l}10 \\
30\end{array}$ & 0.5 & $\begin{array}{l}0.0734 \\
0.1270\end{array}$ & $\begin{array}{l}0.0654 \\
0.1176\end{array}$ & $\begin{array}{l}0.0822 \\
0.1752\end{array}$ & $\begin{array}{l}0.0716 \\
0.1388\end{array}$ & $\begin{array}{l}0.1056 \\
0.2410\end{array}$ & $\begin{array}{l}0.0872 \\
0.1660\end{array}$ \\
\hline & $\begin{array}{l}10 \\
30\end{array}$ & 0.9 & $\begin{array}{l}0.1646 \\
0.4208\end{array}$ & $\begin{array}{l}0.1304 \\
0.3382\end{array}$ & $\begin{array}{l}0.2358 \\
0.5892\end{array}$ & $\begin{array}{l}0.1718 \\
0.4198\end{array}$ & $\begin{array}{l}0.3202 \\
0.7412\end{array}$ & $\begin{array}{l}0.1996 \\
0.4934\end{array}$ \\
\hline & $\begin{array}{l}10 \\
30\end{array}$ & -0.3 & $\begin{array}{l}0.0678 \\
0.0984\end{array}$ & $\begin{array}{l}0.0632 \\
0.0974\end{array}$ & $\begin{array}{l}0.0820 \\
0.1348\end{array}$ & $\begin{array}{l}0.0708 \\
0.1166\end{array}$ & $\begin{array}{l}0.0952 \\
0.1872\end{array}$ & $\begin{array}{l}0.0704 \\
0.1372\end{array}$ \\
\hline & $\begin{array}{l}10 \\
30\end{array}$ & -0.5 & $\begin{array}{l}0.0710 \\
0.0972\end{array}$ & $\begin{array}{l}0.0612 \\
0.0902\end{array}$ & $\begin{array}{l}0.0822 \\
0.1262\end{array}$ & $\begin{array}{l}0.0670 \\
0.0986\end{array}$ & $\begin{array}{l}0.0978 \\
0.1584\end{array}$ & $\begin{array}{l}0.0690 \\
0.1176\end{array}$ \\
\hline & $\begin{array}{l}10 \\
30\end{array}$ & -0.9 & $\begin{array}{l}0.0600 \\
0.0700\end{array}$ & $\begin{array}{l}0.0632 \\
0.0796\end{array}$ & $\begin{array}{l}0.0712 \\
0.1048\end{array}$ & $\begin{array}{l}0.0644 \\
0.0868\end{array}$ & $\begin{array}{l}0.0868 \\
0.1244\end{array}$ & $\begin{array}{l}0.0636 \\
0.0976\end{array}$ \\
\hline \multirow{6}{*}{0.5} & $\begin{array}{l}10 \\
30\end{array}$ & 0.3 & $\begin{array}{l}0.1110 \\
0.2542\end{array}$ & $\begin{array}{l}0.0962 \\
0.2090\end{array}$ & $\begin{array}{l}0.1572 \\
0.3820\end{array}$ & $\begin{array}{l}0.1254 \\
0.2752\end{array}$ & $\begin{array}{l}0.2046 \\
0.5298\end{array}$ & $\begin{array}{l}0.1412 \\
0.3278\end{array}$ \\
\hline & $\begin{array}{l}10 \\
30\end{array}$ & 0.5 & $\begin{array}{l}0.1302 \\
0.3032\end{array}$ & $\begin{array}{l}0.1106 \\
0.2494\end{array}$ & $\begin{array}{l}0.1624 \\
0.4384\end{array}$ & $\begin{array}{l}0.1392 \\
0.3202\end{array}$ & $\begin{array}{l}0.2310 \\
0.5996\end{array}$ & $\begin{array}{l}0.1610 \\
0.3800\end{array}$ \\
\hline & $\begin{array}{l}10 \\
30\end{array}$ & 0.9 & $\begin{array}{l}0.3686 \\
0.8328\end{array}$ & $\begin{array}{l}0.2992 \\
0.7268\end{array}$ & $\begin{array}{l}0.5452 \\
0.9416\end{array}$ & $\begin{array}{l}0.3886 \\
0.8334\end{array}$ & $\begin{array}{l}0.6986 \\
0.9940\end{array}$ & $\begin{array}{l}0.4680 \\
0.9132\end{array}$ \\
\hline & $\begin{array}{l}10 \\
30\end{array}$ & -0.3 & $\begin{array}{l}0.1052 \\
0.2160\end{array}$ & $\begin{array}{l}0.0906 \\
0.1808\end{array}$ & $\begin{array}{l}0.1418 \\
0.3176\end{array}$ & $\begin{array}{l}0.1030 \\
0.2388\end{array}$ & $\begin{array}{l}0.1858 \\
0.4512\end{array}$ & $\begin{array}{l}0.1232 \\
0.2746\end{array}$ \\
\hline & $\begin{array}{l}10 \\
30\end{array}$ & -0.5 & $\begin{array}{l}0.0994 \\
0.2038\end{array}$ & $\begin{array}{l}0.0860 \\
0.1722\end{array}$ & $\begin{array}{l}0.1198 \\
0.2862\end{array}$ & $\begin{array}{l}0.1042 \\
0.2156\end{array}$ & $\begin{array}{l}0.1672 \\
0.3982\end{array}$ & $\begin{array}{l}0.1074 \\
0.2540\end{array}$ \\
\hline & $\begin{array}{l}10 \\
30\end{array}$ & -0.9 & $\begin{array}{l}0.0898 \\
0.1576\end{array}$ & $\begin{array}{l}0.0732 \\
0.1264\end{array}$ & $\begin{array}{l}0.1136 \\
0.2104\end{array}$ & $\begin{array}{l}0.0840 \\
0.1584\end{array}$ & $\begin{array}{l}0.1356 \\
0.2846\end{array}$ & $\begin{array}{l}0.0868 \\
0.1894\end{array}$ \\
\hline \multirow{6}{*}{0.8} & $\begin{array}{l}10 \\
30\end{array}$ & 0.3 & $\begin{array}{l}0.2284 \\
0.5696\end{array}$ & $\begin{array}{l}0.1740 \\
0.4502\end{array}$ & $\begin{array}{l}0.3376 \\
0.7706\end{array}$ & $\begin{array}{l}0.2292 \\
0.5844\end{array}$ & $\begin{array}{l}0.4594 \\
0.9030\end{array}$ & $\begin{array}{l}0.2786 \\
0.6686\end{array}$ \\
\hline & $\begin{array}{l}10 \\
30\end{array}$ & 0.5 & $\begin{array}{l}0.2532 \\
0.6528\end{array}$ & $\begin{array}{l}0.2168 \\
0.5156\end{array}$ & $\begin{array}{l}0.4026 \\
0.8540\end{array}$ & $\begin{array}{l}0.2698 \\
0.6666\end{array}$ & $\begin{array}{l}0.5294 \\
0.9504\end{array}$ & $\begin{array}{l}0.3240 \\
0.7588\end{array}$ \\
\hline & $\begin{array}{l}10 \\
30\end{array}$ & 0.9 & $\begin{array}{l}0.7560 \\
0.9974\end{array}$ & $\begin{array}{l}0.6286 \\
0.9780\end{array}$ & $\begin{array}{l}0.9012 \\
0.9998\end{array}$ & $\begin{array}{l}0.7558 \\
0.9974\end{array}$ & $\begin{array}{l}0.9754 \\
1.0000\end{array}$ & $\begin{array}{l}0.8588 \\
0.9998\end{array}$ \\
\hline & $\begin{array}{l}10 \\
30\end{array}$ & -0.3 & $\begin{array}{l}0.2102 \\
0.5040\end{array}$ & $\begin{array}{l}0.1606 \\
0.4006\end{array}$ & $\begin{array}{l}0.2896 \\
0.7026\end{array}$ & $\begin{array}{l}0.1914 \\
0.5116\end{array}$ & $\begin{array}{l}0.3982 \\
0.8592\end{array}$ & $\begin{array}{l}0.2436 \\
0.5908\end{array}$ \\
\hline & $\begin{array}{l}10 \\
30\end{array}$ & -0.5 & $\begin{array}{l}0.1778 \\
0.4564\end{array}$ & $\begin{array}{l}0.1536 \\
0.3614\end{array}$ & $\begin{array}{l}0.2596 \\
0.6416\end{array}$ & $\begin{array}{l}0.1884 \\
0.4528\end{array}$ & $\begin{array}{l}0.3702 \\
0.8024\end{array}$ & $\begin{array}{l}0.2160 \\
0.5472\end{array}$ \\
\hline & $\begin{array}{l}10 \\
30\end{array}$ & -0.9 & $\begin{array}{l}0.1434 \\
0.3166\end{array}$ & $\begin{array}{l}0.1158 \\
0.2624\end{array}$ & $\begin{array}{l}0.2038 \\
0.4810\end{array}$ & $\begin{array}{l}0.1440 \\
0.3320\end{array}$ & $\begin{array}{l}0.2654 \\
0.6356\end{array}$ & $\begin{array}{l}0.1618 \\
0.3968\end{array}$ \\
\hline
\end{tabular}

RSS = ranked set sampling; SRS = simple random sampling.

Table 3: Empirical nominal value $(\alpha=0.05)$ for three interventional groups

\begin{tabular}{|c|c|c|c|c|c|c|c|}
\hline \multirow{3}{*}{$r$} & \multirow{3}{*}{$\rho$} & \multicolumn{6}{|c|}{$H_{0}: \gamma_{1}=\gamma_{2}=\gamma_{3}$} \\
\hline & & \multicolumn{2}{|c|}{$m=3$} & \multicolumn{2}{|c|}{$m=4$} & \multicolumn{2}{|c|}{$m=5$} \\
\hline & & RSS & SRS & RSS & SRS & RSS & SRS \\
\hline 10 & \multirow[b]{2}{*}{0.3} & 0.0434 & 0.0516 & 0.0476 & 0.0506 & 0.0414 & 0.0452 \\
\hline 30 & & 0.0392 & 0.0508 & 0.0346 & 0.0498 & 0.0374 & 0.0478 \\
\hline 10 & \multirow{2}{*}{0.5} & 0.0388 & 0.0520 & 0.0430 & 0.0530 & 0.0414 & 0.0484 \\
\hline 30 & & 0.0346 & 0.0520 & 0.0334 & 0.0542 & 0.0360 & 0.0466 \\
\hline 10 & \multirow{2}{*}{0.9} & 0.0444 & 0.0490 & 0.0480 & 0.0502 & 0.0464 & 0.0484 \\
\hline 30 & & 0.0338 & 0.0488 & 0.0422 & 0.0526 & 0.0436 & 0.0474 \\
\hline 10 & \multirow[b]{2}{*}{-0.3} & 0.0542 & 0.0538 & 0.0544 & 0.0520 & 0.0422 & 0.0472 \\
\hline 30 & & 0.0378 & 0.0486 & 0.0396 & 0.0492 & 0.0412 & 0.0504 \\
\hline 10 & \multirow{2}{*}{-0.5} & 0.0512 & 0.0516 & 0.0484 & 0.0532 & 0.0512 & 0.0568 \\
\hline 30 & & 0.0380 & 0.0488 & 0.0332 & 0.0502 & 0.0374 & 0.0484 \\
\hline 10 & \multirow{2}{*}{-0.9} & 0.0516 & 0.0524 & 0.0504 & 0.0588 & 0.0498 & 0.0556 \\
\hline 30 & & 0.0364 & 0.0460 & 0.0402 & 0.0510 & 0.0364 & 0.0524 \\
\hline
\end{tabular}

RSS = ranked set sampling; SRS = simple random sampling. 
Table 4: Empirical power for testing three interventional groups with mean different $\left(d_{1}, d_{2}\right)$

\begin{tabular}{|c|c|c|c|c|c|c|c|c|}
\hline \multirow{2}{*}{$d$} & \multirow{2}{*}{$r$} & \multirow{2}{*}{$\rho$} & \multicolumn{2}{|c|}{$m=3$} & \multicolumn{2}{|c|}{$m=4$} & \multicolumn{2}{|c|}{$m=5$} \\
\hline & & & RSS & SRS & RSS & SRS & RSS & SRS \\
\hline \multirow{12}{*}{$0.1 / 0.3$} & 10 & \multirow[b]{2}{*}{0.3} & 0.0757 & 0.0706 & 0.0780 & 0.0680 & 0.0834 & 0.0686 \\
\hline & 30 & & 0.0762 & 0.0904 & 0.1154 & 0.0110 & 0.1586 & 0.1154 \\
\hline & 10 & \multirow{2}{*}{0.5} & 0.0700 & 0.0700 & 0.0750 & 0.0740 & 0.0928 & 0.0680 \\
\hline & 30 & & 0.0978 & 0.0970 & 0.1360 & 0.1068 & 0.1902 & 0.1420 \\
\hline & 10 & \multirow[b]{2}{*}{0.9} & 0.1500 & 0.0800 & 0.1670 & 0.1280 & 0.2564 & 0.1746 \\
\hline & 30 & & 0.3290 & 0.2768 & 0.4828 & 0.3398 & 0.6772 & 0.4242 \\
\hline & 10 & \multirow{2}{*}{-0.3} & 0.0846 & 0.0506 & 0.0940 & 0.0610 & 0.1078 & 0.0612 \\
\hline & 30 & & 0.0640 & 0.0632 & 0.0784 & 0.0634 & 0.0876 & 0.0694 \\
\hline & 10 & \multirow[b]{2}{*}{-0.5} & 0.0778 & 0.0810 & 0.1010 & 0.0986 & 0.1346 & 0.1014 \\
\hline & 30 & & 0.0648 & 0.0614 & 0.0790 & 0.0560 & 0.0894 & 0.0736 \\
\hline & 10 & \multirow{2}{*}{-0.9} & 0.0772 & 0.0758 & 0.0950 & 0.0798 & 0.1220 & 0.1054 \\
\hline & 30 & & 0.0614 & 0.0550 & 0.0638 & 0.0574 & 0.0814 & 0.0616 \\
\hline \multirow{12}{*}{$0.5 / 0.3$} & 10 & \multirow[b]{2}{*}{0.3} & 0.0908 & 0.0828 & 0.1130 & 0.0900 & 0.1600 & 0.1104 \\
\hline & 30 & & 0.1880 & 0.1492 & 0.2900 & 0.2072 & 0.4064 & 0.2524 \\
\hline & 10 & \multirow[b]{2}{*}{0.5} & 0.1000 & 0.0845 & 0.1430 & 0.1020 & 0.1844 & 0.1264 \\
\hline & 30 & & 0.2196 & 0.1936 & 0.3360 & 0.2464 & 0.4952 & 0.3024 \\
\hline & 10 & \multirow[b]{2}{*}{0.9} & 0.2842 & 0.2312 & 0.4310 & 0.2920 & 0.6056 & 0.3744 \\
\hline & 30 & & 0.7516 & 0.6260 & 0.9248 & 0.7600 & 0.9844 & 0.8508 \\
\hline & 10 & \multirow[b]{2}{*}{-0.3} & 0.0876 & 0.0740 & 0.1068 & 0.0920 & 0.1660 & 0.0968 \\
\hline & 30 & & 0.1684 & 0.1440 & 0.2384 & 0.1948 & 0.3564 & 0.2088 \\
\hline & 10 & \multirow[b]{2}{*}{-0.5} & 0.0876 & 0.0844 & 0.1156 & 0.0832 & 0.1396 & 0.0916 \\
\hline & 30 & & 0.1360 & 0.1288 & 0.2193 & 0.1705 & 0.3160 & 0.1912 \\
\hline & 10 & \multirow[b]{2}{*}{-0.9} & 0.0725 & 0.0712 & 0.0992 & 0.0668 & 0.1096 & 0.0856 \\
\hline & 30 & & 0.1060 & 0.0944 & 0.1616 & 0.1308 & 0.2208 & 0.1496 \\
\hline \multirow{12}{*}{$0.3 / 1.0$} & 10 & \multirow{2}{*}{0.3} & 0.2746 & 0.2054 & 0.3994 & 0.2654 & 0.5568 & 0.3434 \\
\hline & 30 & & 0.6797 & 0.5803 & 0.8820 & 0.6986 & 0.9714 & 0.7903 \\
\hline & 10 & \multirow[b]{2}{*}{0.5} & 0.3034 & 0.2340 & 0.4700 & 0.3180 & 0.6420 & 0.4074 \\
\hline & 30 & & 0.7920 & 0.6371 & 0.9406 & 0.7811 & 0.9894 & 0.8726 \\
\hline & 10 & \multirow{2}{*}{0.9} & 0.8606 & 0.7517 & 0.9771 & 0.8886 & 0.9974 & 0.9388 \\
\hline & 30 & & 1.0000 & 0.9991 & 1.0000 & 0.9997 & 1.0000 & 1.0000 \\
\hline & 10 & \multirow[b]{2}{*}{-0.3} & 0.2731 & 0.1917 & 0.4277 & 0.2737 & 0.5737 & 0.3340 \\
\hline & 30 & & 0.7048 & 0.5497 & 0.8982 & 0.6951 & 0.9717 & 0.7903 \\
\hline & 10 & \multirow[b]{2}{*}{-0.5} & 0.3440 & 0.2668 & 0.5034 & 0.3280 & 0.6777 & 0.3886 \\
\hline & 30 & & 0.7866 & 0.6460 & 0.9411 & 0.7831 & 0.9883 & 0.8731 \\
\hline & 10 & & 0.8837 & 0.7674 & 0.9743 & 0.8891 & 0.9980 & 0.9354 \\
\hline & 30 & -0.9 & 1.0000 & 0.9980 & 1.0000 & 1.0000 & 1.0000 & 1.0000 \\
\hline
\end{tabular}

RSS = ranked set sampling; SRS = simple random sampling.

Table 5: Empirical power for testing two treatments effect with mean treatment groups different $(d)$ using ranked set sampling for the proposed approach compared with an ordinary regression approach

\begin{tabular}{|c|c|c|c|c|c|c|}
\hline \multirow[b]{2}{*}{$d$} & \multirow[b]{2}{*}{$r$} & \multirow[b]{2}{*}{$\rho$} & \multicolumn{2}{|c|}{ Proposed approach } & \multicolumn{2}{|c|}{ Regression approach } \\
\hline & & & $m=3$ & $m=4$ & $m=3$ & $m=4$ \\
\hline \multirow[b]{2}{*}{0.0} & 10 & 0.3 & 0.0410 & 0.0474 & 0.0250 & 0.0250 \\
\hline & 10 & 0.9 & 0.0388 & 0.0496 & 0.0350 & 0.0310 \\
\hline \multirow[b]{2}{*}{0.3} & 10 & 0.3 & 0.0692 & 0.0892 & 0.0580 & 0.0840 \\
\hline & 10 & 0.9 & 0.1646 & 0.2358 & 0.1260 & 0.2280 \\
\hline \multirow[b]{2}{*}{0.5} & 10 & 0.3 & 0.1110 & 0.1572 & 0.1060 & 0.1330 \\
\hline & 10 & 0.9 & 0.3686 & 0.5452 & 0.3550 & 0.5340 \\
\hline
\end{tabular}

this experiment, conducted by the Department of Psychology, in collaboration with the College of Public Health, Department of Biostatistics. This study was approved by the institutional review board at Georgia Southern University (IRB number: H116063).

Approximately 250 undergraduate students at Georgia Southern University were recruited for the study. All participants over the age of 18 and enrolled in a psychology course, were eligible for 
the intervention experiment. In the first phase of data collection, the 250 recruited undergraduate students completed a short series of surveys that included: the demographic questionnaire and the Ryff psychological well-being scale-short form (RPWS-SF). The RPWS-SF is an 18-item measure of psychological well-being, defined as a state of contentment marked by self-acceptance, positive relations with others, autonomy, environmental mastery, purpose in life, and personal growth (Ryff, 1989). Participants rate their agreement on each item on a 6-point ordinal scale, with responses ranging from strongly agree to strongly disagree. Total scores are summative and range from 18 to 108, with higher scores indicating greater levels psychological well-being. Participants were directed to an invitation page after completing the questionnaires and asked to participate in phase II of the study.

A total of 183 participants who expressed interest in completing the second phase of the study, served as the participant pool for phase II. The selection of participants for phase II was determined by variation in well-being score ranks, which was the baseline auxiliary covariate. There are only two experimental groups in the study (the gratitude-based emotional uplifting intervention group and the control group); therefore, only 60 students were selected for phase II based on the modified RSS protocol in Section 2. We select set size of RSS $m=3$ because we have a small pool of students to select from to include in phase II in the study.

Selected participants for the gratitude-based emotional uplifting intervention group and the control group were asked to complete a questionnaire about their mood in order to measure positive and negative emotions (positive and negative affect schedule, PANAS). The PANAS is a brief, 20-item measure of positive and negative mood states. Respondents were asked to rate their current emotional state based upon 20-unique, emotional prompts. Participants were asked to rate their mood on a scale of 1 to a 100. Participants were asked to complete a narrative-based writing task after completing the first set. The writing task asked participants to reflect on personal experiences with goal completion/achievement.

After completing the second set of emotion questionnaires, individuals assigned to the emotional uplifting intervention group were asked to extend positive emotions through a gratitude-based task. This exercise helped individuals mindfully extend and enhance positive emotions associated with a goal completion/achievement. However, the second group of participants in the control group, were not directed to complete an assigned task and were asked to wait in a private room until the Research Assistant instructed them further. Completion of both tasks was expected to last approximately 20 minutes. All participants were asked to complete a series of self-report measures after completing one of the assigned tasks. These measures will include the PANAS, the coping self-efficacy scale (a valid measure of coping self-efficacy), the Connor-Davidson Resilience Scale (a valid measure of resilience), the Oxford Happiness Questionnaire (a valid measure of happiness), and a demographic form. It was expected that participants would be able to complete the entire process in approximately 70-75 minutes.

This experiment was designed to investigate the relations between positive experiences and contributing higher levels of happiness, well-being, and life satisfaction. The uplifting intervention strategy group was assessed by measuring the reported positive mood and compared with the control group. As an illustration to our proposed approach we focused on testing the hypothesis that "the increase in mood would be experienced more significantly in participants who were selected into the intervention group compared to the control group."

The comparison in the intervention groups was accomplished by using ANCOVA models. Out of the selected 60 students for the phase II intervention, 45 students (23 students in the uplifting group and 22 students in the control group) responded and completed the second set of questioners. 
Table 6: Analysis of covariance in a multiple regression model

\begin{tabular}{ccccc}
\hline \hline Parameter & Estimate & Standard error & $t$-value & $p$-value \\
\hline Intercept & 724.81 & 357.42 & 2.03 & 0.0489 \\
Uplifting & 210.16 & 77.29 & 2.72 & 0.0095 \\
Wellbeing total & -3.98 & 4.31 & -0.92 & 0.3604 \\
\hline \hline
\end{tabular}

For testing the hypothesis, a multiple regression approach in an ANCOVA model was used to investigate positive mood contribution in both the uplifting intervention group and the control group (Table 6). It would be concluded that there were highly significant differences in final positive mood between the intervention and the control groups, $p$-value $=0.0095$. Note that, the expected increase of positive mood scores is about 210.16 for participants in the uplifting group (intervention group) compared to the control group. This indicates that the participants with higher total wellbeing scores had less benefit in getting a positive mood since the estimated coefficient of wellbeing scores total had a negative sign. This also indicates that using more structural sampling procedure (such as RSS) based on the wellbeing scores total was beneficial in this study since RSS balanced the disparity of this covariate in the two groups compared. However, characteristic covariates such as age, marital status, race, and academic class were not significant factors.

Moreover, with a sample size of 45 students, the estimated power for the analysis of covariance is 0.73. However, the estimates for the statistical power of analysis for a sample size of 60,30 students in each group, is more than 0.8 . Therefore, RSS is an effective methodology for subject selection to improve the outcome means comparisons of intervention groups with small sample sizes.

\section{Concluding remarks}

RSS has been applied to problems in ecological and environmental science as well as adopted for use in experimental and epidemiological applications. The work presented in this paper is to help frame a modified approach of RSS for an efficient application to experimental studies that can compare $L$ groups.

RSS is beneficial, especially when the measurements of interest are expensive and/or not easy to obtain. Along with the experimental outcome, other information such as auxiliary covariates are highly correlated to the outcome of interest and can be used to estimate the population mean by reducing the required sample size. In addition to the previously discussed intensive literature this paper suggests that those baseline covariates can be used to improve the inference of intervention comparisons when the measures of interest outcome is difficult to obtain in terms of cost and time.

We proposed a modified RSS scheme to examine the treatment effects between two or more experimental groups that use one available auxiliary covariate known to be correlated with the variable of interest. Under the proposed protocol, subjects can be selected for an experimental study based on the ranked auxiliary covariate and randomly allocated into the experimental groups. Therefore, the modified RSS suggested in this paper is shown to be an effective approach to select more structured and representative samples based on the most available and cost efficient auxiliary covariates.

An ANCOVA model was proposed for making inferences with the use of continuous auxiliary covariate data. WLS was used to estimate the model's parameters when the observed values of variability differ over the predictor values of the ranked subjects, where weights are determined using the variance of the order statistics. Therefore, the WLS for the auxiliary covariate parameter $\hat{\beta}_{\mathrm{RSS}}$ and the groups indicator variable's parameter $\hat{\gamma}_{\mathrm{RSS}}$ are unbiased estimators for the population estimators $\beta$ and $\gamma$ and have lower variances when compared to using SRS, respectively. However, the modi- 
fied RSS method proposed is a more powerful testing procedure to compare different groups using ANCOVA model than using SRS. The strength of this modified sampling scheme is that it is a costeffective method for some experimental studies. Section 4 illustrates the proposed modified RSS in an experimental study conducted by the Department of Psychology and the College of Public Health at Georgia Southern University. The method of the modified RSS was used to select subjects into the experimental study that were randomly assigned to a gratitude-based emotional uplifting intervention group and a control group.

In conclusion, the modified RSS is an effective methodology for subject selection to improve the comparison of intervention groups with small sample sizes. However, there is a significant limitation in the proposed sampling scheme as it may not be valid in clinical trials, where it is considered unethical to discard fully measured units (Samawi and Vogel, 2015).

\section{References}

Barreto MCM and Barnett V (1999). Best linear unbiased estimators for the simple linear regression model using ranked set sampling, Environmental and Ecological Statistics, 6, 119-133.

Chen Z, Bai Z, and Sinha BK (2004). Ranked Set Sampling: Theory and Applications, Springer, New York.

Chen Z, Liu J, Shen L, and Wang YG (2008). General ranked set sampling for efficient treatment comparisons, Statistica Sinica, 18, 91-104.

Donner A and Zou GY (2007). Methods for the statistical analysis of binary data in split-mouth designs with baseline measurements, Statistics In Medicine, 26, 3476-3486.

Egger J, Graham PJ, Carter CM, Gumley D, and Soothill JF (1985). Controlled trial of oligoantigenic treatment in the hyperkinetic syndrome, Lancet, 325, 540-545.

McIntyre GA (1952). A method for unbiased selective sampling, using ranked sets, Australian Journal of Agricultural Research, 3, 385-390.

Muttlak HA (1996). Estimation of parameters in a multiple regression model using rank set sampling, Journal of Information and Optimization Sciences, 17, 521-533.

Özdemir YA and Esin AA (2007). Parameter estimation in multiple linear regression models using ranked set sampling, Communications Series Al Mathematics $\mathcal{E}$ Statistics, 56, 7-20.

Patil GP, Sinha AK, and Taillie C (1993). Relative precision of ranked set sampling: a comparison with the regression estimator, Environmetrics, 4, 399-412.

Rohatgi VK and Ehsanes Saleh AKMD (2001). An Introduction to Probability and Statistics, John Wiley \& Sons, New York.

Ryff CD (1989). Happiness is everything, or is it?: explorations on the meaning of psychological well-being, Journal of Personality \& Social Psychology, 57, 1069-1081.

Samawi HM and Ababneh FM (2001). On regression analysis using ranked set sample, Journal of Statistical Research (JSR), 35, 93-105.

Samawi H and Vogel R (2015). On ranked set sampling variation and its applications to public health research. In DG Chen and J Wilson (Eds), Innovative Statistical Methods for Public Health Data (pp. 291-313).

Samawi HM and Abu-Dayyeh W (2002). On regression analysis with random regressors using ranked samples, International Journal of Information and Management Sciences, 13, 19-36.

Sandelowski M (2000). Focus on research methods: combining qualitative and quantitative sampling, data collection, and analysis techniques in mixed-method studies, Research in Nursing \& Health, 23, 246-255. 
Stokes SL (1977). Ranked set sampling with concomitant variables, Communications in Statistics Theory \& Methods, 6, 1207-1211.

Tsiatis AA, Davidian M, Zhang M, and Lu X (2008). Covariate adjustment for two-sample treatment comparisons in randomized clinical trials: a principled yet flexible approach, Statistics in Medicine, 27, 4658-4677.

Zhang M, Tsiatis AA, and Davidian M (2008). Improving efficiency of inferences in randomized clinical trials using auxiliary covariates, Biometrics, 64, 707-715.

Received December 26, 2016; Revised February 4, 2017; Accepted March 11, 2017 